\title{
Une démarche d'ergonomie de la formation
}

Un projet pilote en formation par simulation d'infirmiers anesthésistes

Ergonomics for Simulation-based Training : A Pilot Design Approach for Anesthetics Nurse Training

\section{Zoya Horcik et Marc Durand}

\section{OpenEdition}

\section{Journals}

Édition électronique

URL : http://journals.openedition.org/activites/2613

DOI : 10.4000/activites.2613

ISSN : $1765-2723$

Éditeur

ARPACT - Association Recherches et Pratiques sur les ACTivités

\section{Référence électronique}

Zoya Horcik et Marc Durand, « Une démarche d'ergonomie de la formation», Activités [En ligne],

8-2 | octobre 2011, mis en ligne le 15 octobre 2011, consulté le 01 mai 2019. URL : http:// journals.openedition.org/activites/2613; DOI : 10.4000/activites.2613

\section{(c) (i) () $\Theta$}

Activités est mis à disposition selon les termes de la licence Creative Commons Attribution - Pas d'Utilisation Commerciale - Pas de Modification 4.0 International. 


\title{
Une démarche d'ergonomie de la formation : Un projet pilote en formation par simulation d'infirmiers anesthésistes
}

\section{Zoya Horcik}

Zoya.Horcik@unige.ch

\section{Marc Durand}

Marc.Durand@unige.ch

\author{
Équipe CRAFT \\ Faculté de Psychologie et de Sciences de l'éducation \\ Université de Genève \\ 40 boulevard du Pont d'Arve - CH 1211 Genève 4
}

\begin{abstract}
SUMMARY
Ergonomics for Simulation-based Training: A Pilot Design Approach for Anesthetics Nurse Training. This paper describes a cooperation between professionals from the Geneva's University Hospitals and our research unit, dealing with a vocational training program for nurses anesthetists using simulation at the SimulHUG Center. Our ergonomic approach of vocational education is presented, based on the assumption of autonomy of the human activity (Theureau, 2004, 2006). This current program counts, at the moment, three stages. After a quick presentation of the training device's first version, we summarize the typical results obtained in Step 2 and areas of possible enrichment planned. The conclusion briefly discusses the nature of relationship between researchers, trainers and principles of cooperation among them.
\end{abstract}

KEYWORDS

Simulation, Nursing, Conception, Training, Fictional experience.

Un intérêt grandissant en formation professionnelle se manifeste pour la prise en compte de l'activité des professionnels et des apprentis (Barbier \& Durand, 2005; Durand \& Filliettaz, 2009). Cet intérêt s'est particulièrement concrétisé dans l'approche de didactique professionnelle (Pastré, 2011) qui peut être envisagée au sens restreint ou au sens large. Au sens restreint, celle-ci vise l'identification de structures conceptuelles caractérisant une tâche ou un travail, c'est-à-dire des invariants cognitifs organisés, afin de documenter les contenus des formations professionnelles (Caens-Martin, Specogna, Delépine, \& Girerd, 2004). Au sens large, la didactique professionnelle peut désigner l'ensemble des approches qui analysent le travail dans une visée de formation. Notre propre programme s'inscrit dans cette définition large (Durand, 2008).

Deux axes ont été explorés dans nos précédentes recherches : celui, déjà décrit, consistant à extraire par l'analyse les composantes ou organisateurs clés du travail réel afin d'alimenter une démarche de transposition didactique, et celui s'appuyant sur les résultats de recherches relatives aux transformations à long terme de l'activité au travail pour concevoir des dispositifs d'accompagnement des trajectoires professionnelles de débutants (Durand, sous presse; Leblanc, Ria, Dieumegard, Serres, \& Durand, 2008; Ria, 2009).

Nous illustrons ici un troisième axe, que nous qualifions d'ergonomie de la formation, à partir de la présentation d'un projet en cours portant sur la formation des infirmiers anesthésistes aux Hôpitaux Universitaires de Genève (HUG) recourant à un simulateur au sein du Centre SimulHUG. Nous 
exploitons dans ce projet, l'idée que la formation est un travail, qui peut faire l'objet d'une approche d'ergonomie visant à la rendre plus efficace, mieux organisée, plus conforme à l'activité réelle des opérateurs.

Béguin (2005) souligne l'aspect dialogique de la démarche de conception ergonomique qui met en relation le concepteur et l'utilisateur dans un mouvement d'aller et retour via le dispositif conçu. Pinski (1992) et Theureau (2004; Theureau \& Jeffroy, 1994) mettent en avant, quant à eux, le caractère cyclique et itératif de cette démarche qui a pour objectif d'analyser l'activité des opérateurs afin d'améliorer les situations de travail en les rendant ajustées au travail réel, plus performantes, confortables, sécures et propices à un développement professionnel. En raison du dynamisme des situations de travail et du caractère indéterminé de l'activité, la démarche ergonomique implique un recours à une itération de l'articulation conception - analyse, afin d'approcher de façon asymptotique de ses objectifs (Theureau \& Jeffroy, 1994).

Notre approche se fonde sur un postulat d'autonomie de l'activité humaine au sens où elle exprime un couplage permanent entre l'acteur et l'environnement, se transformant constamment du fait de sa dynamique propre et qui intègre des perturbations significatives pour l'acteur en provenance de son environnement (Theureau, 2004, 2006). Cette dynamique s'auto-construit pas à pas, de sorte que l'activité est envisagée comme une totalité auto-organisée faisant émerger ses formes signifiantes qui ne sont pas réductibles à, ou explicables par, des causes externes à elle (bien qu'elle soit perturbée par une partie des événements se produisant dans l'environnement). Cette conception amène à considérer que les significations des événements et des transformations de l'environnement ne sont pas fixées, pré-données ou transmises, mais construites ou appropriées par les acteurs. Consécutivement à ce premier élément, l'activité ne peut donc pas être instruite ou commandée par l'intervention d'autres acteurs tels que des ergonomes ou des formateurs, ou des transformations de l'environnement. Le couplage acteur - environnement n'est donc pas conceptualisé comme un flux de représentations ou d'ajustements aux contraintes adaptatives provenant de l'environnement, mais comme une émergence dynamique, organisante et organisée, qui spécifie son unité, l'entretient et la transforme dans le temps.

Nous avons donc adopté une démarche itérative, dans une perspective visant l'enrichissement du travail de formation par la simulation d'infirmiers anesthésistes. Cette démarche est encore en cours et compte à ce jour trois étapes principales : Étape 1 : conception et mise en œuvre d'une Version 1 du dispositif de formation par les formateurs en l'absence d'intervention de la part des chercheurs; Étape 2 : analyse de l'activité des formateurs et des formés au sein de la Version 1 du dispositif; Étape 3 (en cours) : transformations et développement de la Version 1 en lien avec les résultats obtenus lors de l'Étape 2.

Après une description triviale de la Version 1 du dispositif et une présentation de l'observatoire de la formation mis en place lors de l'Étape 2, nous résumons les résultats typiques obtenus lors de l'Étape 2, et les axes d'enrichissement de la formation envisagés. La conclusion traite brièvement de la nature des rapports entre chercheurs et formateurs et des principes de cette coopération.

\section{1.- Dispositif et observatoire de la formation}

Le projet a été initié il y a 24 mois, et a impliqué deux chercheurs de l'équipe de l'Université de Genève, trois formateurs des Hôpitaux Universitaires de Genève (HUG), et une dizaine d'infirmiers anesthésistes en formation spécialisée d'anesthésistes par la simulation. Notre choix de nous centrer sur cette formation répondait à deux critères principaux : il s'agissait d'un secteur en pointe des HUG qui s'appuyait sur une bonne maîtrise de la simulation (les formateurs étaient formés et expérimentés et avaient en leur possession une banque de scénarios finalisés); d'autre part, le dispositif en place accueillait différents programmes de formation permettant en raison de cela de suivre des cohortes de formés pendant une année, et de compléter ces observations par des recueils ponctuels auprès 
d'autres formations (le présent article rend compte de l'activité de trois de ces formés qui ont été l'objet d'un suivi longitudinal, mais les résultats valent pour l'ensemble des observations réalisées).

\section{1.- Participants}

Le projet a principalement mobilisé trois types d'acteurs : trois formateurs expérimentés $(A, B, C)$ en matière de simulation; trois infirmiers en formation (A, B, C), inscrits dans le cursus de formation spécialisée d'infirmiers anesthésistes. Tous ont été volontaires pour participer au projet qui a été validé par le comité d'éthique des HUG. Il a également impliqué deux chercheurs de l'Université de Genève, spécialistes en analyse du travail et formation professionnelle, avec deux formes d'implication complémentaires : au contact des formations par une présence assidue sur le terrain et un travail systématique sur les données de la recherche pour l'une, par une présence épisodique sur le terrain et un travail systématique sur les données pour l'autre. Les deux ont été impliqués dans les interventions, formations et interactions diverses qui jalonnent la conduite du projet.

\section{2.- Le Centre SimulHUG}

La formation a eu lieu au Centre SimulHUG qui disposait d'une salle d'opération contenant le matériel nécessaire à la procédure d'anesthésie, ainsi que du matériel de simulation : un mannequin robotisé de pleine échelle permettant diverses interventions telles que des intubations, poses de sondes, injections de médicaments; un scope simulant les paramètres vitaux d'un patient; du matériel d'enregistrement (caméras et micros). Il comprenait également une salle de contrôle d'où les formateurs pilotaient le déroulement des scénarios et observaient les participants, grâce à l'écran transmettant les images filmées par les caméras. Enfin, une salle de briefing/débriefing était équipée d'un vidéoprojecteur et d'un grand écran permettant la diffusion des enregistrements (Figure 1).
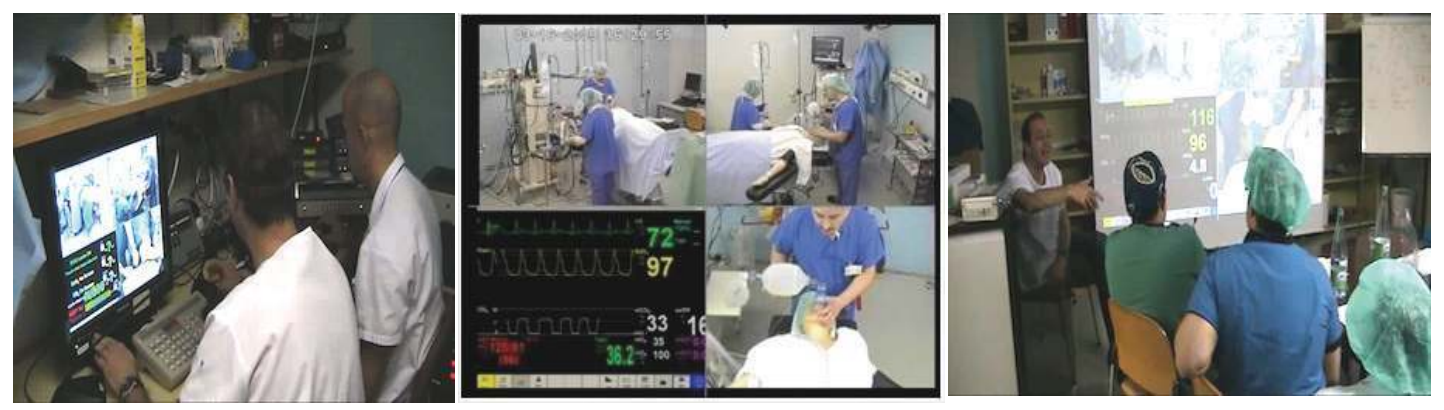

Figure 1 : Vues de la salle de contrôle, de la salle d'opération et de la salle de débriefing

Figure 1: View of the control room, the operating theatre and the debriefing room

\section{3.- Les séances de formation}

Lors des séances de formation à l'anesthésie, les formés ont été confrontés à des scénarios conçus par les formateurs, qui se basaient sur des cas réels issus de la littérature scientifique ou de l'expérience professionnelle des infirmiers anesthésistes et des formateurs. Ils étaient considérés par eux comme emblématiques du travail d'anesthésiste, et mettaient en jeu des situations normales, incidentelles, incidentelles/accidentelles, et accidentelles telles que des réveils par induction simple, rapide ou sans complications, des régurgitations pendant l'induction, des complications inattendues durant le réveil du patient, etc. Ces cas emblématiques étaient traduits sous forme de séquences qui spécifiaient les actions et les paramètres correspondants du mannequin. La tâche des formés était de collaborer en vue d'anesthésier et de réveiller le patient/mannequin sans danger et en respectant les procédures instituées. 
Une séance de formation comportait généralement trois phases.

Lors de la Phase 1 dite de briefing, les formateurs présentaient aux formés la salle d'opération, les fonctionnalités du mannequin, leur délivraient les consignes concernant le scénario de la simulation en les invitant notamment à s'engager dans des séquences simulant l'endormissement et le réveil de patients, comme ils le feraient en situation réelle.

Lors de la Phase 2, un scénario était réalisé par les formés, et piloté et supervisé par les formateurs. L'évolution des informations relatives au mannequin était commandée par un programme informatique préétabli par les formateurs qui pouvaient également reprendre la main durant son déroulement. Le mannequin ne réagissait pas directement à l'action des formés, mais à celle des formateurs qui le pilotaient en fonction de ce qui se passait dans la salle d'opération. Les scénarios utilisés étaient consacrés à l'endormissement et au réveil d'un patient dans des conditions et selon des protocoles variés. Ils étaient généralement proposés selon un ordre croissant de complexité au cours d'une séance et d'une séance à l'autre. Ils comportaient également un jeu de rôle au sens où les formés étaient invités à assurer des fonctions diverses, pendant la simulation, autres que celles d'infirmier anesthésiste : interne, chirurgien, aide-soignant et infirmier instrumentiste.

Lors de la Phase 3, un débriefing avait lieu durant lequel formateurs et formés commentaient et analysaient les événements liés au scénario à partir du film enregistré.

\section{4.- L'observatoire de la formation}

L'observatoire mis en place visait à identifier : a) les principes effectivement opérants dans la formation dans la Version 1 du dispositif, b) les traits principaux de l'activité des formateurs et des formés au sein de la Version 1 du dispositif. Cet observatoire impliquait une présence assidue de chercheurs dans le Centre SimulHUG, collaborant avec les participants à la formation dans le cadre d'une enquête coopérative tenue par un partage d'objectifs relatifs à une amélioration de l'efficacité de ce dispositif de formation et une meilleure connaissance des phénomènes s'y produisant (Durand \& Horcik, sous presse). Les données recueillies l'ont été lors a) d'observations et prises de notes ethnographiques, d'enregistrements audio et vidéo, b) de séances d'auto-confrontation, c) d'entretiens informels, individuels et collectifs avec les participants portant sur les résultats empiriques et des informations-débats et les options de formation, et d) de séminaires et journées d'étude.

Les données d'observation étaient constituées des enregistrements effectués par le système vidéo du centre de simulation et d'enregistrements, faits par les chercheurs, à l'aide d'une caméra vidéo des actions et verbalisations réalisées en salle de contrôle et de débriefing.

Les séances d'auto-confrontation (Theureau, 2004, 2010) ont été réalisées entre une semaine et un mois après les observations de terrain. Des sélections étaient opérées dans les enregistrements. Les épisodes soumis aux participants avaient été repérés par les chercheurs comme potentiellement intéressants pour documenter les spécificités de la simulation et les liens avec les situations de travail réel. Ces séquences pouvaient être étendues ou réduites en fonction des commentaires et des souhaits des professionnels interrogés. Les séances, individuelles, avaient lieu aux HUG; elles commençaient par une séquence de remise en situation dynamique des participants, qui étaient placés face aux enregistrements vidéo de leur activité lors des briefings, simulations proprement dites et débriefings. Les acteurs étaient incités à revivre les actions et événements et à exprimer leur expérience lorsque ceuxci s'étaient produits. Cette expression se faisait lors du défilement de l'enregistrement, et d'arrêts de la vidéo (à l'initiative des chercheurs ou des participants). Les participants étaient invités à décrire, montrer et raconter leur activité, et à éviter les justifications et explications pour accéder aux descriptions par les acteurs des composantes de leur expérience lors des épisodes enregistrés. Les questions et relances des chercheurs se focalisaient sur la description des savoirs mobilisés dans l'action (e.g. Vous pensez à quelque chose de précis à ce moment-là?), les interprétations (e.g. Vous vous dîtes quoi là ?), les validations et invalidations (e.g. Cela confirme-t-il ce que vous pensiez?), les généra- 
lisations (e.g. Est-ce souvent ainsi pour vous?). Elles avaient pour objectif : a) de centrer l'attention des acteurs sur un événement particulier : "Qu'est-ce qui se passe à ce moment-là? »); b) les inciter à préciser leur description (par exemple : «Pouvez-vous m'en dire plus là-dessus? » "Qu'est-ce qui vous fait réagir ainsi? », «Vous percevez quelque chose en particulier?»); et c) d'obtenir des informations supplémentaires ("Que ressentez-vous à ce moment-là? », "Vous cherchez à faire quelque chose de particulier? »). Les entretiens ont été enregistrés intégralement à l'aide d'une caméra vidéo.

Les entretiens informels concernaient les formateurs et portaient sur la simulation et sa place dans le programme de formation général de l'hôpital, ils étaient l'occasion de centrer le questionnement sur les objectifs poursuivis, les convictions pédagogiques des formateurs, leurs sources d'information et complétaient l'étude de différents scénarios, feuilles médicales utilisées pour la simulation, transmission d'articles et de recherches sur lesquels étaient basés les scénarios.

Les séminaires et journées d'étude réunissaient les chercheurs et formateurs et pouvaient être élargis à des spécialistes de la formation (chercheurs et formateurs) non impliqués dans le projet. Ils consistaient en des exposés systématiques et des discussions improvisées, qui portaient sur la présentation, la discussion et l'interprétation des résultats, et l'imagination collective de principes complémentaires fondant la Version 1 du dispositif.

\section{5.- Traitement et analyse des données}

Le traitement et l'analyse ont cherché a) à caractériser la conception qui sous-tendait la formation mise en place telle qu'elle résultait du travail de conception des formateurs, en lien avec l'analyse de la formation effectivement réalisée et de l'activité des formateurs et des formés dans ce dispositif; b) à identifier d'éventuels aspects minorés par les formateurs, non perçus ou non contrôlés par eux et paraissant ou non-prometteurs d'apprentissage.

L'analyse de l'activité des participants à la formation était centrale. Le traitement a été réalisé en trois phases :

- La Phase 1 consistait en la retranscription verbatim du corpus de vidéos en protocoles à deux volets mettant en vis-à-vis des épisodes issus des données d'observation et les auto-confrontations correspondantes.

- La Phase 2 consistait en la reconstitution des cours d'action des acteurs et a fait l'objet d'une analyse selon la démarche canonique dans ce cadre théorique (Theureau, 2004, 2006, 2010). Elle a fait l'objet d'une présentation systématique (Horcik, Savoldelli, \& Durand, soumis). Le traitement visait l'identification de composantes de l'activité, d'actions ou de séquences typiques chez les participants. Étaient considérés comme typiques des aspects de l'activité apparaissant sous une forme jugée identique avec une fréquence élevée (plusieurs fois chez chaque acteur à chaque séance de formation, chez plusieurs acteurs), et évaluée comme emblématique de l'activité en question par les participants (directement lors des auto-confrontations et des séminaires) ainsi que par les chercheurs après codage et traitement de l'ensemble du corpus.

Le caractère de typicité d'une composante de l'activité a été évalué en rapport avec la théorie initialement proposée par Rosch, Simpson et Miller (1976) : est typique un élément estimé comme le meilleur représentant du corpus constituant une catégorie plus large. Cet élément est présenté dans les parties 2 et 3 de cet article à partir d'une modélisation locale qui condense les traits de plusieurs occurrences, et plus souvent à partir de la description d'une occurrence réelle considérée comme typique.

- La Phase 3 a consisté en une mise en correspondance des résultats issus de l'analyse de l'activité, avec les traits typiques du dispositif de formation. Des éléments complémentaires ont été pris en considération provenant des entretiens informels et des séminaires, qui ont permis d'aboutir à la formulation d'énoncés généraux et abstraits caractérisant ce que nous considérons comme un modèle pragmatique de la formation. 


\section{Le Modèle de Formation par Simulation des Formateurs}

À chaque fois que cela paraît possible, les formateurs fondent leurs décisions sur des résultats de recherches. Inspirés des démarches evidence based en médecine, ces résultats proviennent a) de revues de littérature ou de méta-analyse relatives à la simulation, b) des recherches ponctuelles relatives à l'effet sur l'apprentissage de tous les facteurs potentiellement actifs (Savoldelli, 2011). Au-delà de ces fondements explicites, il nous a été possible de caractériser un modèle de la formation, moins clairement énoncé, que nous qualifions en trois points : fidélité/réalisme, instruction/prescription, et réflexivité.

\subsection{Réalisme et fidélité au travail}

Ce qui est recherché par la formation est une fidélité à l'environnement et aux situations réelles de travail. Cette quête de réalisme est cohérente avec les recherches qui montrent qu'un intérêt majeur de la simulation tient à ce qu'elle permet de solliciter une activité similaire à l'activité professionnelle de référence, mais dans des conditions spécifiques telles que la suspension ou l'euphémisation des conséquences de l'action qui autorise les erreurs, la production de situations exceptionnelles, les possibilités de varier différents paramètres intéressants pour l'apprentissage, le jeu possible avec une réversibilité du temps, etc. (Bradley, 2006; Dieckmann, Manser, Wehner, \& Rall, 2007; Gaba, Howard, Fish, Smith, \& Sowb, 2001 ; Pastré \& Rabardel, 2005).

Les scénarios conçus par les formateurs cherchent à reprendre de manière fidèle des situations pouvant avoir lieu en salle d'opération en sélectionnant des séquences de l'activité visées au cours desquelles l'action des anesthésistes est fréquente ou cruciale. Il ne s'agit pas ici d'un découpage portant sur une action spécifique (comme intuber en conditions difficiles, passer la main à un collègue), mais d'une délimitation d'empans temporels (endormissement, surveillance, réveil) par rapport à l'activité cible, au cours desquels tous les aspects de l'activité des anesthésistes sont mobilisés.

Lors de la Phase 1 (briefing) les formateurs cherchent à présenter le simulateur aux formés en les laissant dans un premier temps, tester les différentes fonctionnalités du mannequin par des essais d'intubation, de pression de masque à oxygène sur le visage, etc. Ils leur indiquent ensuite l'emplacement des caméras et les éléments qui diffèrent des blocs opératoires dans lesquels ils travaillent habituellement. Ces deux premiers éléments visent à réduire un possible effet de déstabilisation des formés au cours du scénario.

En complément, dans cet environnement réel ou au moins réaliste, les consignes des formateurs insistent sur l'exigence de fidélité au travail réel. L'Épisode Type 1 est typique des consignes délivrées lors des briefings, qui insistent sur le fait que ce qui est attendu des formés est un travail identique au travail réel.

Formateur A : C'est un monsieur qui vous attend pour être endormi (...) qui a été vu par un anesthésiste. Il a une feuille qui est prête. Il y a le plateau de médicament qui est prêt. Vous discutez avec lui et l'équipe chirurgicale va vous donner des choses... C'est tout ce que vous avez comme renseignements. Je pense que vous devriez pouvoir vous en sortir.

Épisode Type 1 illustré par un extrait du briefing en salle d'opération Typical Episode 1 illustrated by an excerpt from briefing in operation room

Ces consignes sont délivrées sous l'hypothèse implicite que l'activité réelle doit être proche de celle prescrite et que celle-ci dépend largement de la précision de la prescription. Et de fait ces consignes sont à l'origine d'une activité similaire ou très proche du travail de la part des formés. Celle-ci se met en place plus ou moins rapidement et facilement. L'Épisode Type 2 issu d'une auto-confrontation d'un formé qui décrivait son activité au début de l'intervention sur le mannequin est typique de cet engagement proche du travail chez les formés.

Formé B : Là, je fonctionne comme d'habitude (...). Rien de particulier, il y a une interaction avec la collègue et puis... Je pense que je commence à être un petit peu dedans parce que je commence 
à regarder le moniteur là en haut (...). Je regarde surtout la courbe de $\mathrm{CO}_{2}$ parce que je ventile mon patient, et donc ça permet de vérifier si la ventilation est efficace.

Épisode Type 2 illustré par un extrait d'entretien d'auto-confrontation à propos du scénario simulé

Typical Episode 2 illustrated by a self-confrontation excerpt about the simulated scenario

Lors de la Phase 2 (effectuation du scénario), le pilotage du mannequin par les formateurs prend en compte les actions des formés, et l'évolution des paramètres vitaux du mannequin, de manière logique ou plausible. Cet ajustement précis des différentes variables vise à respecter les paramètres hémodynamiques du mannequin de manière plausible par rapport à une situation de travail réelle similaire. Il est aussi fréquent que des perturbations soient introduites pendant les scénarios (i.e. interventions d'un formateur/chirurgien qui demande aux formés de se dépêcher, pose des questions intempestives, fait du bruit dans la salle).

Lors de la Phase 3 (débriefing) les formateurs cherchent essentiellement à se focaliser sur les éléments du travail-cible et relativement peu sur l'activité des formés en simulation. L'enjeu est principalement de les amener à parler des situations de travail réel à travers le scénario simulé.

\section{2.- Interventions normatives et instructives des formateurs}

Les consignes des formateurs sont destinées à faire surgir chez les formés une activité proche du travail réel; et leur activité s'apparente à celle des ergonomes organisant le travail d'autrui. Mais le dispositif ainsi conçu n'est pas seulement ergonomique : il est aussi formatif au sens où il vise une transformation de l'activité des formés et des acquisitions en rapport avec leur travail. Pour ce faire les interventions observées peuvent être qualifiées de prescriptives et instructives.

Les débriefings sont explicitement conçus comme les moments clés de la formation (Savoldelli, 2011). Ils sont structurés par anticipation, principalement sur la base des observations faites par les formateurs durant la Phase 2 de simulation. Ils se présentent comme une succession de séquences ayant un rapport avec les événements du scénario, mais recelant des possibilités de généralisation. Ces séquences consistent en des interactions riches et complexes entre les formateurs et les formés, et comportent une orientation normative essentielle, particulièrement lorsque les formés sont débutants. Des thèmes non prévus peuvent apparaître en lien avec la dynamique des interactions et les intérêts des formés; mais, avec une fréquence très élevée, ce sont les formateurs qui ont la main sur le déroulement du débriefing au cours duquel ils privilégient certains thèmes puis introduisent des éléments de généralité portant sur les avantages ou inconvénients de telle ou telle action. Bien qu'elles présentent des ajustements ou des variations, les séquences d'activité des formateurs lors de ces séquences de débriefing adoptent la forme typique présentée dans le Tableau 1.

Faire défiler la vidéo jusqu'à ce qui est considéré comme la fin d’un épisode ayant été repéré lors de la simulation

Arrêter le défilement de la vidéo pour solliciter les formés à proposer un commentaire de l'épisode et/ou une évaluation des actions individuelles et collectives

Animer et relancer la discussion au sujet de l'épisode en question

Porter un jugement sur la pertinence des actions des uns et des autres

Rappeler les normes et procédures (lorsqu'il y en a) régissant l'épisode en question

Apporter des éléments de connaissance clinique et/ou scientifique relatifs à l'épisode en question

Envisager un ou des épisode(s) virtuels différents par certaines caractéristiques et ce qu'il aurait alors convenu de faire

S'assurer de la compréhension, de l'accord et/ou de l'adhésion des formés

Activer le défilement de la vidéo jusqu'à l'épisode suivant

Etc.

Tableau 1 : Séquence typique de l'activité des formateurs lors des débriefings

Table 1: Typical sequence of trainers's activity during debriefings 
L'Épisode Type 3 (Interactions formateur-formés lors un débriefing) et 4 (auto-confrontation d'un formateur à l'enregistrement d'un débriefing portant sur un autre épisode que le précédent sont des occurrences partielles de cette séquence typique.

Formée A : Au début quand on a regardé la feuille du patient, je ne savais pas non plus si je devais mettre une sonde nasogastrique... Comme je ne connais pas....

Formateur A : Dans quel cas ça aurait été intéressant? Quel élément t'aurait permis de dire : «OK il faut mettre une sonde nasogastrique »?

Formés A et B : Des vomissements?

Formateur A : Oui, voilà.

Formée A : On aurait dû le demander au patient?

Formateur B : En général c'est fait avant... Il n'y a pas tellement de recommandations par rapport à ça.

Épisode Type 3 illustré par un extrait concernant un débriefing

Typical Episode 3 illustrated by an excerpt about the debriefing

Formateur A : Je voulais saisir au vol ce qui allait arriver. Là en préambule de cette séquence, je voulais juste décortiquer l'écran qu'on a ici. Le scope... avec les paramètres du patient qui sont bons en fait! Je leur dis : « ce n'est pas cette situation que vous aurez tout le temps. S'il se passe autre chose qu'est ce que vous devrez faire? » Je leur donne aussi les autres possibles et je passe à autre chose.

Épisode Type 4 illustré par un extrait d'entretien d'auto-confrontation au sujet du débriefing portant sur un autre épisode que le précédent

Typical Episode 4 illustrated by a self-confrontation excerpt about another debriefing

\section{3.- Une formation réflexive}

Le dispositif SimulHUG est basé sur une séparation/articulation entre le scénario simulé et le débriefing. Le scénario est censé permettre une expérimentation par les formés de séquences préalablement identifiées (réveil, endormissement), et fournir aux formateurs des éléments utilisables dans les débriefings. Au début de chaque séquence-type décrite ci-dessus, ces débriefings présentent une partie interactive impliquant la réflexivité des formés. Ces débuts de séquences n'ont pas seulement comme fonction de fournir un matériau ou un thème pour un rappel des normes et un enseignement : elles actualisent l'intention des formateurs de faire réfléchir les formés à ce qu'ils ont fait. Ceux-ci sont incités à faire le récit de ce qui s'est passé dans la salle d'opération, et éventuellement, par des séries de questions de plus en plus précises, à prendre conscience ou rendre explicites de leurs points de vue sur divers aspects de la situation. Cela aboutit à des reconstructions collectives ouvrant sur une interprétation commune de la situation, et sur une interprétation de la part du formateur, qui synthétise et clôt cette enquête locale (Épisode Type 5).

Formateur A : Je cherche à ce qu'ils revisitent un peu leur histoire. Ils ont l'histoire devant les yeux en plus... À voir tout ce qui a concouru à faire que B n'arrive pas à faire quelque chose de facile (...). Quels sont les différents éléments qu'on peut faire bouger? Quelles sont les variables qu'on peut mobiliser? Qu'on peut changer pour que l'action devienne réussie? Et puis chacun apporte son truc. Moi j'ai vu ça, j'ai vu ça... Et puis finalement on peut tout regrouper... Et puis à la fin je vais résumer en disant « voilà les différentes choses. ». C'est ce que je me donne comme rôle.

Épisode Type 5 illustré par un extrait d'entretien d'auto-confrontation au sujet du débriefing

Typical Episode 5 illustrated by a self-confrontation excerpt about the debriefing

Une reconstruction et une mise en intrigue sont opérées à partir de l'analyse de la prestation des formés, qui servent de support à la réflexion pendant la séquence et au-delà (Rudolph, Simon, Raemer, \& Eppich, 2008; Savoldelli, Naik, Park, Joo, Chow, \& Hamstra, 2006). Un aspect saillant de ces épisodes est qu'ils doivent favoriser les prises de conscience de la part des formés à travers la 
reconstruction de l'activité simulée et l'explicitation des normes qui la cadrent, au moyen des apports dirigés par les formateurs. Ces enquêtes collectives aboutissent souvent à l'énonciation de conseils pour l'activité cible (Épisode Type 6).

Formé B : Ouais... La table n'est pas à la bonne hauteur. Je suis en train de penser que si elle avait été un peu plus haute, j'aurais été plus confortable, là j'ai dû fléchir les jambes pour me mettre à niveau. (...)

Formateur A : Ouais t'étais pas confortable hein?

Formé B : Non

Formateur A : Comme quoi l'ergonomie a son importance... Surtout quand ça va mal. Alors le conseil c'est : quand ça va bien, ben... pensez-y déjà.

Épisode Type 6 : illustré par un extrait concernant un débriefing

Typical Episode 6: illustrated by an excerpt about the debriefing

\section{3.- Vers l'enrichissement du modèle de formation}

Cette partie concerne notre contribution à la conception d'une Version 2 du dispositif de formation, informée par les résultats empiriques obtenus à l'étape précédente du projet ergonomique. Il s'agit d'une étape de coopération-conseil avec les formateurs en charge de la formation. Nous nous constituons en aides potentielles pour ces formateurs, dans la mesure où nous sommes détenteurs d'une expertise générale en matière d'analyse du travail et de la formation professionnelle, et où nous avons pu identifier des aspects inconnus, masqués ou négligés dans le dispositif de formation du Centre SimulHUG.

Notre contribution vise à favoriser la concrétisation de trois principes de conception susceptibles d'enrichir la Version 1 du dispositif. Ces principes peuvent être résumés ainsi : a) compléter la recherche de fidélité des environnements de formation par une centration sur l'activité des formés, b) cet intérêt explicite et instrumenté pour l'activité et l'expérience des formés conduisant aussi à prendre au sérieux la composante mimétique et fictionnelle de leur activité dans la formation, et c) engager une conception proscriptive (et non prescriptive) de l'intervention formative.

\section{1.- La recherche de fidélité formelle au travail complétée par une centration sur l'activité des formés}

Nos résultats soutiennent l'idée d'un intérêt à la recherche d'une ressemblance entre l'activité en formation et au travail plutôt que celle d'une ressemblance entre les environnements de formation et de travail. La première ne va pas sans la seconde, mais comme l'a déjà montré la recherche en didactique professionnelle (Caens-Martin et al. 2004; Nyssen, 2005), il est plus prometteur d'apprentissage de concevoir des simulateurs sollicitant une activité ressemblant à celle du travail, que des simulateurs ressemblant physiquement aux environnements de travail. Et, si un effort est à accomplir sur ce point, il doit moins concerner la recherche d'une fidélité formelle que le développement de simulateurs susceptibles de solliciter tout ou partie des composantes typiques de l'activité de référence, pour laquelle les acteurs doivent être formés (Boucheix \& Chanteclair, 1999; CaensMartin, 1999). Deux étapes supplémentaires de recherche sont apparues nécessaires à ce point du travail de conception. La première concerne l'identification de ces composantes critiques du travail d'infirmier anesthésiste (qui sont probablement pour une large part insérées dans les scénarios imaginés par les formateurs, mais sur la base de leur expérience professionnelle d'infirmiers, et sans méthode contrôlée et explicitée pour couvrir le cœur de ce métier et ses subtilités). La deuxième concerne le repérage non pas seulement d'aspects critiques du travail d'infirmier anesthésiste, tels qu'ils peuvent ressortir de l'analyse du travail de professionnels expérimentés, mais des obstacles et situations critiques rencontrés par les débutants pour lesquels des dispositifs ajustés à leurs difficultés spécifiques sont à concevoir. Nous nous inspirons en cela des travaux déjà entrepris dans d'autres 
domaines professionnels tels que l'enseignement en milieu difficile (Ria, 2009).

Ceci ne suppose pas de révoquer la recherche de fidélité du simulateur par rapport au travail, mais de distribuer l'effort collectif dans diverses directions qui pourraient consister à a) se satisfaire du degré de fidélité au travail actuellement atteint, b) obtenir des assurances quant à la centralité des contenus de formation insérés dans les scénarios proposés aux formés par les formateurs, c) compléter la palette de scénarios actuellement disponibles (comportant une composante de programmation du mannequin), par la détermination de cas portant non pas sur la stricte reconduction de séquences significatives ou archétypes du travail d'infirmier anesthésiste, mais sur des problèmes récurrents et significatifs rencontrés par les novices (les enregistrements disponibles de toutes les séquences de formation réalisées à ce jour dans le centre SimulHUG constituent de ce point de vue un matériau de choix).

\section{2.- Un intérêt pour l'activité des formés conduisant notamment à prendre au sérieux la composante mimétique et fictionnelle de leur activité}

Le réalisme qui préside à la conception de la formation accrédite l'idée d'une activité simulée proche du travail réel et conforme à ce que souhaitent les formateurs. Cela les incite à ne prendre en compte que des éléments ayant trait au travail lui-même. Or, l'analyse montre une activité spécifique, riche et complexe chez les formés dans la simulation.

Ceux-ci sont, en permanence, engagés dans une interprétation des éléments de la situation et tâchent en même temps de déterminer si ces événements appartiennent à la simulation ou au scénario effectivement prévu par les formateurs. Les événements inédits, impromptus, les complications de la situation peuvent relever d'erreurs de programmation du mannequin, d'oubli de matériel et de conséquences de leurs propres actions. Et bien que la possibilité de pièges ne soit jamais annoncée lors des briefings, les formés sont convaincus qu'ils vont y être confrontés; ils tentent alors de les identifier pendant la simulation et focalisent une partie de leurs attentes sur leur survenue. Cette anticipation d'événements problématiques, critiques ou surprenants est une composante notable de l'expérience des formés qui se livrent à ce qu'on peut qualifier de surinterprétations (Épisode Type 7).

Formé B : «Il y a toujours une petite méfiance quand même, parce qu'on sait qu'on est en simulation. On ne va pas nous faire travailler forcément la situation idéale; les formateurs vont essayer de nous faire émerger des situations potentiellement problématiques (...). Donc on est toujours en train de guetter et de se dire : «Où est-ce qu'ils vont faire survenir quelque chose, un incident ou une particularité?»

Épisode Type 7 illustré par un extrait d'entretien d'auto-confrontation à propos du scénario simulé

Typical Episode 7 illustrated by a self-confrontation excerpt about the simulated scenario

Comme l'indique l'Épisode Type 8, ces interrogations sur la présence ou non de pièges pourraient constituer un matériau pour une exploitation approfondie en prévision de situations au cours desquelles se poseraient des questions d'anticipation et de décision. Cet épisode a impliqué le Formé B, lors d'un délestage vers sa collègue $\mathrm{A}$ à la suite d'un problème d'intubation non prévu par les formateurs. Le Formé B, qui opérait alors à la tête du mannequin, a supposé que plusieurs événements incidentels imprévus et liés à ses propres difficultés d'intubation et d'interprétation du scénario, avaient été préparés par les formateurs (lesquels n'avaient effectivement anticipé qu'une urgence liée à une régurgitation factice intervenue précédemment).

Formé B : (...) je me suis dit : « est-ce qu'ils ont modifié une partie du mannequin pour faire une intubation plus difficile? »(...) parce que je crois que mes deux collègues étaient passés avant, tout s'était bien passé, Cormack 1 (critère servant à évaluer la difficulté, liée à l'anatomie des patients, de l'intubation; le Niveau 1 caractérise une intubation «facile»), et puis je me dis : «je n'y arrive pas ». Et puis on me présente un patient qui cause pendant l'oxygénation; ensuite on me fait le coup 
du reflux gastro-œsophagien, et puis là maintenant je me dis : « je n'y arrive pas, ils ont dû modifier une partie du mannequin pour nous faire faire une intubation difficile? ».

Épisode Type 8 illustré par un extrait d'entretien d'auto-confrontation à propos du scénario simulé

Typical Episode 8 illustrated by a self-confrontation excerpt about the simulated scenario

Ne pouvant résoudre cette difficulté d'intubation, il a décidé de passer la main à sa collègue (Formée A) qui est parvenue sans problème à intuber le mannequin. Il a ensuite observé attentivement sa collègue (Épisode Type 9).

Formé B : Je me dis, on va voir si effectivement y'a un piège ou pas. Là il y'a ma collègue qui va le faire; et puis, soit ça va pas jouer et elle ne va pas y arriver non plus et puis dans ce cas, il y a eu une embûche de plus, une difficulté de plus qu'ils ont mis sur le parcours. Donc je me dis : « Soit ! Réfléchissons déjà à l'avance, si ça marche pas qu'est-ce qu'on fait?»

Épisode Type 9 illustré par un extrait d'entretien d'auto-confrontation à propos du scénario simulé

Typical Episode 9 illustrated by a self-confrontation excerpt about the simulated scenario

Par ailleurs, les activités des formés, si elles sont articulées dans l'action, obéissent aussi à des dynamiques relativement indépendantes qui peuvent conduire à des interprétations contradictoires de la situation, et des engagements des formés différents. Ceci n'est pris en compte par les formateurs, ni dans le pilotage de la séquence, ni dans le débriefing. L'Épisode Type 10 illustre une telle divergence.

La Formée A qui opérait à la tête du patient pendant une procédure de réveil problématique (laryngospasme à l'extubation), était intensément immergée dans la situation durant toute la durée de la simulation; elle était focalisée sur les paramètres vitaux du mannequin qui chutaient. Après avoir éprouvé des difficultés à le ventiler, et s'être demandé si cette difficulté était voulue par les formateurs, elle s'est rapidement convaincue qu'elle devait l'intuber pour permettre un retour à la normale de ses signes vitaux. Elle cherchait avant tout à réagir comme elle le ferait en situation réelle.

Formée A : Le souci, c'est que j'étais focalisée sur la saturation qui baissait. (...) Et en fait je n'ai pas pensé à la fréquence cardiaque, mais dans mon esprit c'était clair : le patient, il fallait l'intuber (...).

Épisode Type 10 illustré par un extrait d'entretien d'auto-confrontation à propos du scénario simulé

Typical Episode 10 illustrated by a self-confrontation excerpt about the simulated scenario

Simultanément, le Formé B était focalisé sur la ventilation du mannequin et cherchait avant tout à comprendre pourquoi il rencontrait des difficultés dans le scénario. Présentant un engagement distant dans la situation, il a inséré dans son diagnostic de la situation l'éventualité d'un piège tendu par les formateurs (Épisode Type 11).

Formé B : Je suis dans l'incompréhension. On ne peut toujours pas le ventiler (le mannequin) malgré nos actions pour lever le laryngospasme (...). Là, je change de matériel (...). Soit il est en spasme complet et on l'intube, soit il n'est pas en spasme complet et on doit absolument essayer de le ventiler. On doit savoir s'il est ventilable ou pas. Donc là, je vais forcer l'air à passer (...). Je veux savoir si notre scénario va jusqu'à l'intubation ou si c'est bon ».

Épisode Type 11 illustré par un extrait d'entretien d'auto-confrontation à propos du scénario simulé

Typical Episode 11 illustrated by a self-confrontation excerpt about the simulated scenario

Cet épisode est typique d'une articulation des activités individuelles seulement superficielle, chacun des acteurs étant engagé dans une activité séparée et peu coordonnée avec celle de l'autre (Horcik, Savoldelli, \& Durand, soumis). Cette dimension collective de l'activité, qui est ici potentiellement 
problématique, n'est pas prise en compte par les formateurs au cours du débriefing. Elle est uniquement envisagée par le Formateur A au cours du pilotage du mannequin comme un élément intéressant, car le Formé $\mathrm{B}$ pense à utiliser un ballon peu habituel pour ce type de procédure; il valide alors cette initiative sans chercher à déconstruire cette prise de décision ou à y revenir, afin de comprendre les choix effectués par les deux formés au cours de la simulation. Or l'un des objectifs affirmés de la formation est la qualité de la coordination collective des actions, notamment dans les phases où la rapidité et la cohérence des décisions individuelles et collectives sont cruciales, et où la communication doit être précise, comme le rappellent fréquemment les formateurs.

Ces résultats indiquent de réelles possibilités d'optimisation de l'impact formatif du dispositif SimulHUG, si l'on prend en compte de façon plus centrale l'activité et l'expérience des formés, et notamment une présence importante de composantes fictionnelle ou mimétique dans cette activité. En effet, si les briefings et les consignes initiales, ainsi que la configuration de l'environnement ont pour visée de déclencher un engagement fictionnel de la part des formés, ce point est considéré comme mineur ou allant de soi dans le dispositif de formation. Le dispositif SimulHUG entretient un rapport subtil de ressemblance et différence avec le travail réel. Ce caractère double conduit un engagement particulier des formés, que nous qualifions à la suite de Schaeffer (1999), d'immersion mimétique. Nous désignons par là un mode d'engagement similaire à celui du travail ou très proche, qui n'est cependant pas celui du travail dans la mesure où les formés ne perdent jamais la conscience d'être dans un cadre de simulation. Cet engagement n'est ni permanent ni stable, il fluctue entre une proximité avec l'activité de référence et une proximité avec l'activité de formation (Épisode Type 12).

Formé B : Là, je commence à m'agiter : je vois cette saturation qui baisse et apparemment A qui n'arrive pas bien à le ventiler, ce patient (...). A me dit très justement « Est-ce qu'on a de quoi le réintuber? » (...). Je lui réponds « oui ok c'est bon », et je n'ai pas pensé qu'ils nous feraient jouer le scénario jusqu'au bout! Je me rends compte après coup que je n'avais rien de prêt. Sur le coup, je me suis dit : «OK on va leur montrer (aux formateurs) qu'on y a bien pensé »...

Épisode Type 12 illustré par un extrait d'entretien d'auto-confrontation à propos du scénario simulé

Typical Episode 12 illustrated by a self-confrontation excerpt about the simulated scenario

Prendre en compte l'activité des formés revêt une réelle importance : certaines interventions des formateurs peuvent par exemple être intempestives et « faire sortir» les formés de cet engagement mimétique. Par ailleurs, par rapport à des promesses d'apprentissage, c'est un aspect qui apparaît essentiel. La richesse de l'apprentissage des formés dépend de la nature de l'immersion mimétique et de ses fluctuations, et notamment de la dynamique très subtile d'oscillation entre prise de distance et recouvrement entre activité simulée et activité réelle. Ces oscillations pourraient être considérées comme telles par les formateurs, notamment lors des consignes, feedback en cours de simulation, recours au récit des formés pendant les débriefings, et plus largement comme objet de formation.

Ces résultats conduisent à une revalorisation de la phase de simulation dans la formation, en raison de sa dimension d'immersion mimétique. La simulation n'est en effet pas seulement un déclencheur ou un épisode collectif servant de référence et de pourvoyeur d'ancrages et de thèmes pour le débriefing auquel est, dans la Version 1, prioritairement alloué le rôle de générateur d'apprentissages. Nos analyses ouvrent sur des hypothèses pratiques contrastées que nous posons comme complémentaires au plan pratique : la simulation dans sa dimension d'immersion mimétique est la base d'une activité fictionnelle au sein de laquelle les formés s'engagent dans un rapport mimétique et ludique, avec leur activité de travail ou avec eux-mêmes au travail, et qui est riche en promesses d'apprentissage. Cela suppose une attention de la part des formateurs, des consignes, des commentaires et une culture de formation davantage centrés sur la le mimétisme et le jeu.

\section{3.- Une intervention proscriptive des formateurs se concrétisant par la}




\section{conception d'espaces d'actions encouragées}

Les résultats de l'analyse de l'activité des formés et des formateurs ont, avec d'autres provenant de domaines professionnels différents, complété un travail conceptuel à partir du postulat d'autonomie de l'activité humaine (Durand, 2008). En résumé ces résultats montrent qu'il se produit dans le cadre de la simulation, des phénomènes bien connus dans le domaine du travail : les opérateurs ne font pas strictement ce qui est attendu d'eux (l'activité réelle diffère de la prescription) et les formateurs doivent pendant les séquences simulées, déployer des rattrapages des événements inattendus et des exploitations opportunistes de ces événements, et pendant les débriefings, des reprises systématiques de la prescription aux différentes étapes de l'anesthésie, notamment durant les phases procéduralisées. Ces observations confirment, selon nous, la valeur heuristique du présupposé sous lequel nous conduisons nos recherches, qui est un présupposé d'autonomie de l'activité. Cela ne revient pas à énoncer que cette activité soit sans contraintes, mais à la considérer comme une émergence, c'està-dire comme auto produisant son ordre et sa signification dans son couplage viable avec l'environnement (lequel comporte les consignes des formateurs, leurs recommandations ou conseils, les éléments factices inhérents au dispositif de simulation, etc.).

Cette hypothèse est incompatible avec ce que Simondon (1989) dénomme un schème hylémorphique, c'est-à-dire la conceptualisation selon laquelle un objet physique, social, symbolique serait obtenu par l'imposition depuis l'extérieur d'une forme à une matière informe ou amorphe. L'alternative à cette hypothèse considérée comme erronée est que c'est la matière qui prend forme dans ses interactions avec le moule, et dans le cas de l'apprentissage c'est l'acteur qui se transforme dans ses interactions avec l'environnement conçu pour lui par les formateurs. C'est donc le formé qui « a la main », ce qui relativise le statut et l'efficacité des consignes délivrées par les formateurs ainsi que l'impact fonctionnel du simulateur et de l'environnement de la simulation.

Les consignes des formateurs et l'ensemble des artefacts déposés dans l'environnement des formés ont un statut paradoxal dans cette conceptualisation fondamentalement constructiviste, dans la mesure où ils visent à prescrire l'activité des formés alors que nous faisons l'hypothèse que cette activité est non instruite et émergente. C'est dire que selon notre interprétation des résultats de l'Étape 2 et nos hypothèses générales relatives à l'activité humaine, la visée de transformation de l'activité des formés par les formateurs ne peut qu'être modeste et douteuse. Elle est modeste au sens où c'est l'acteur lui-même qui, in fine, spécifie celles qui, des perturbations introduites dans son environnement, sont significatives pour lui, et seront intégrées dans son activité. Elle est douteuse au sens où les formateurs n'ont jamais de certitude quant à ce qui va être significatif pour le formé, va perturber son activité et occasionner des rééquilibrations majorantes. Et il ne peut qu'avoir un espoir (et non une certitude) d'impact formatif.

C'est pourquoi nous considérons les environnements de formation conçus dans le cadre de SimulHUG comme des espaces d'action encouragées (Durand, 2008), c'est-à-dire des ensembles délimitant des actions possibles (et encouragées) et d'autres impossibles (et découragées). L'intervention formatrice est proscriptive dans la mesure où elle agit directement sur ce qui ne doit ou ne peut être réalisé, et indirectement sur ce qui peut ou doit l'être en définissant par la négative (en lien avec ce processus de découragement) un espace protégé au sein duquel peuvent émerger ou s'auto-produire des actions espérées prometteuses d'apprentissages.

Ce principe porte une posture modeste des formateurs qui réduit leur action à la définition précise de limites ou normes d'acceptabilité non discutables ou non négociables, et de suggestions et recommandations dont le mode opératoire est celui de la perturbation espérée signifiante pour les formés et déclencheuse de rééquilibrations compatibles avec leurs normes propres. L'action de formation est indirecte (elle agit sur l'environnement des formés et non sur leur système cognitif, leurs représentations ou leur activité) et proscriptive (elle délimite indirectement par des interdits des ensembles d'actions possibles). Elle débouche ainsi, par le détour et l'inversion, sur des promesses d'apprentissage. 


\section{Conclusion}

Le rôle des chercheurs est non pas de proposer une alternative au dispositif actuel de formation, qui est évalué comme innovant et d'une incontestable efficacité, mais de rendre possible et acceptable la prise au sérieux de l'activité des utilisateurs dans la conception et la mise en œuvre de la formation. À ce jour, en plus de l'activité de recherche empirique proprement dite, notre coopération avec les formateurs a pris diverses formes : a) présentation des résultats obtenus et discussion de nos interprétations au cours d'une formation de formateurs centrée sur l'analyse de l'activité, b) familiarisation des formés avec les démarches d'analyse de l'activité et présentation des gains escomptés d'une prise au sérieux de l'activité pour la conception, et c) ouverture de possibles respectant la logique de la formation mise en place, mais en en étendant et diversifiant les leviers d'action.

Nos interactions avec les formateurs, pour des raisons tant épistémologiques qu'éthiques, respectent le postulat d'autonomie de leur activité énoncé plus haut. Elles sont donc conduites dans la perspective de construction d'espaces cognitifs mutuellement partagés et co-construits, et non pas de la formulation ponctuelle de recommandations surplombantes (Durand \& Horcik, sous presse).

L'instauration d'une culture de l'activité au sein du Centre SimulHUG est apparue en cours de projet comme son enjeu central, non pas parce que les formateurs se désintéressent habituellement de cette notion ou de cet objet, mais parce que cela demeure, comme c'est souvent le cas chez les professionnels de la formation, une sorte d'allant de soi ou une évidence non questionnée. Outre la formation de formateur évoquée plus haut, cette instauration pourrait s'opérer par des initiatives localisées et convergentes, telles que a) l'organisation au sein du dispositif de séquences permettant d'accéder à l'expérience des acteurs par un recours à des méthodes telles que l'auto-confrontation ou d'autres modalités systématiques d'accès à la subjectivité des acteurs, issues de l'analyse du travail, b) l'exploitation, en complément des débriefings actuels à visée réflexive et transmissive, du matériau issu de ces méthodes pour alimenter un accès raisonné et non évaluatif des formés à leur propre activité, la compréhension de celle d'autrui, et la construction de référentiels partagés et c) la construction d'un habitus de l'invention chez les formateurs simultanément à leur appropriation des méthodes et concepts de l'ergonomie de conception centrée sur l'activité, leur permettant de prendre en charge de façon autonome les démarches innovantes dans leur travail de formateurs.

\section{RÉFÉRENCES}

Barbier, J.-M., \& Durand, M. (2005). Les rapports sujets - activités - environnements. Paris: PUF.

Béguin, P. (2005). Concevoir pour les genèses professionnelles. In P. Rabardel, \& P. Pastré (Eds.), Modèles du sujet pour la conception : dialectiques, activités, développement (pp. 31-52). Toulouse: Octarès.

Boucheix, J.-M., \& Chanteclair, A. (1999). Analyse de l'activité, cognition et construction de situations d'apprentissages : Le cas des conducteurs de grues à tour. Éducation Permanente, 139, 115-141.

Bradley, P. (2006). The history of simulation in medical education and possible future directions. Medical Education, 40, 254-262.

Caens-Martin, S. (1999). Une approche de la structure conceptuelle d'une activité agricole : La taille de la vigne. Éducation Permanente, 139, 99-114.

Caens-Martin, S., Specogna, A., Delépine, L., \& Girerd, S. (2004). Un simulateur pour répondre aux besoins de formation sur la taille de la vigne. Sciences et Technologies de l'Information et de la Communication pour l'Éducation et la Formation, 11. En ligne, http://sticef.univ-lemans.fr/num/vol2004/delepine-02/ sticef_2004_delepine_02.pdf.

Dieckmann, P., Manser, T., Wehner, T., \& Rall, M. (2007). Reality and fiction cues in medical patient simulation: An interview study with anesthesiologists. Journal of Cognitive Engineering and Decision Making, l(2), 148-168. 
Durand, M. (2008). Un programme de recherche technologique en formation des adultes. Éducation \& Didactique, 2(3), 97-121.

Durand, M. (sous presse). Self-constructed activity, work analysis, and occupational training: An approach to learning objects for adults. In P. Jarvis (Ed.), The Routledge international handbook on learning. London: Routledge.

Durand, M., \& Filliettaz, L. (2009). Travail et formation des adultes. Paris: PUF.

Durand, M., \& Horcik, Z. (sous presse). Pour une autre alliance du savoir et de l'action : L'invention d'espaces pratiques de travail-formation-recherches mutuellement fécondes. In F. Yvon \& M. Durand (Eds.), Analyser l'activité en éducation et en formation. Bruxelles: De Boeck.

Gaba, D.-M., Howard, S.-K., Fish, K.-J., Smith, B.-E., \& Sowb, Y.-A. (2001). Simulation-based training in anaesthesia crisis resource management (ACRM): A decade of experience. Simulation \& Gaming, 32(2), 175-193.

Horcik, Z., Savoldelli, G., \& Durand, M. (soumis). L'expérience fictionnelle d'infirmiers anesthésistes en formation professionnelle sur un simulateur de pleine échelle.

Leblanc, S., Ria, L., Dieumegard, G., Serres, G., \& Durand, M. (2008). Concevoir des dispositifs de formation professionnelle des enseignants à partir de l'analyse de l'activité dans une approche énactive. @ctivités, 5(1), 58-78.

Nyssen, A.-S. (2005). Simulateurs dans le domaine de l'anesthésie. Études et réflexions sur les notions de validité et de fidélité. In P. Pastré. \& P. Rabardel (Eds.), Apprendre par la simulation. De l'analyse du travail aux apprentissages professionnels (pp. 269-283). Toulouse: Octarès.

Pastré, P. (2011). La didactique professionnelle. Paris: PUF.

Pastré, P., \& Rabardel, P. (2005). Apprendre par la simulation. De l'analyse du travail aux apprentissages professionnels. Toulouse: Octarès.

Pinsky, L. (1992). Concevoir pour l'action et la communication. Essais d'ergonomie cognitive. Berne : Peter Lang.

Ria, L. (2009). Concevoir des formations centrées sur l'analyse de l'activité : le cas de l'enseignement en milieux difficiles. In M. Durand \& L. Filliettaz (Eds.), Travail et formation des adultes (pp. 217-243). Paris : PUF.

Rosch, E., Simpson, C., \& Miller, R. S. (1976). Structural bases of typicality effects. Journal of Experimental Psychology: Human Perception and Performance, 2, 491-502.

Rudolph, J.-W., Simon, R., Raemer D.-B. \& Eppich, W.-J. (2008). Debriefing as formative assessment: Closing performance gaps in medical education. Academic Emergency Medicine, 15(11), 1010-1016.

Savoldelli, G. (2011). «Pas de simulation sans débriefing! ». Simulation et formation : journée d'étude et d'échanges RIFT, équipe CRAFT, Genève, 27 mai.2011.

Savoldelli, G., Naik, V.-N., Park, J., Joo, H.-S., Chow, R., \& Hamstra, S.-J. (2006). Value of debriefing during simulated crisis management: Oral versus video-assisted oral feedback. Anesthesiology, 105(2), 279-285.

Schaeffer, J.-M. (1999). Pourquoi la fiction? Paris: Seuil.

Simondon, G. (1989). Du mode d'existence des objets techniques. Paris: Aubier.

Theureau, J. (2004). Le cours d'action. Méthode élémentaire. Toulouse: Octarès.

Theureau, J. (2006). Le cours d'action. Méthode développée. Toulouse: Octarès.

Theureau, J. (2010). Les entretiens d'auto-confrontation et de remise en situation par les traces matérielles et le programme de recherche « cours d'action ». Revue d'Anthropologie des Connaissances, 4(2), 287-322.

Theureau, J., Jeffroy, F. (1994). Ergonomie des situations informatisées : La conception centrée sur le cours d'action de l'utilisateur. Toulouse: Octarès. 


\begin{abstract}
RÉSUMÉ
Cet article rend compte d'une démarche de rechercheentre des professionnels des Hôpitaux Universitaires de Genève et notre unité de recherche, portant sur une formation par simulation d'infirmiers anesthésistes actuellement en cours dans le Centre SimulHUG. Il décrit une démarche d'ergonomie de la formation professionnelle centrée sur l'activité et conduite sous un postulat d'autonomie de l'activité humaine (Theureau, 2004, 2006). Cette démarche, actuellement en cours, compte pour l'instant, trois étapes. Après une description triviale de la Version 1 du dispositif et une présentation de l'observatoire de la formation mis en place lors de l'Étape 2, nous résumons les résultats typiques obtenus lors de l'Étape 2, et les axes d'enrichissement de la formation envisagés. La conclusion traite brièvement de la nature des rapports entre chercheurs et formateurs et des principes de cette coopération.
\end{abstract}

Mots CLÉS

Simulation, Soins infirmiers, Conception, Expérience fictionnelle, Formation.

\title{
RÉFÉRENCEMENT
}

Horcik, Z., \& Durand M. (2011). Une démarche d'ergonomie de la formation : Un projet pilote en formation par simulation d'infirmiers anesthésistes. Activités, 8(2), pp. 173-188, http://www.activites.org/v8n2/v8n2. pdf

Article soumis le 28 avril 2011, accepté pour publication le 29 août 2011 